\title{
The Pressure- and Temperature-Induced Wetting Transitions in the Binary Water + Ethylene Glycol Monoisobutyl Ether Mixture
}

\author{
Chih-Kang Wu and Li-Jen Chen* \\ Department of Chemical Engineering, National Taiwan University, Taipei 10617, Taiwan
}

Received: February 21, 2006; In Final Form: April 27, 2006

\begin{abstract}
A homemade pendent drop/bubble tensiometer is applied to perform the surface/interfacial tension measurements for the binary water + ethylene glycol monoisobutyl ether (iso- $\mathrm{C}_{4} \mathrm{E}_{1}$ ) mixture over the temperature range from 25 to $150{ }^{\circ} \mathrm{C}$ and over the pressure range up to 100 bar. The symbol $\mathrm{C}_{i} \mathrm{E}_{j}$ is the abbreviation of a nonionic polyoxyethylene alcohol $\mathrm{C}_{i} \mathrm{H}_{2 i+1}\left(\mathrm{OCH}_{2} \mathrm{CH}_{2}\right)_{j} \mathrm{OH}$. The wetting behavior of the iso$\mathrm{C}_{4} \mathrm{E}_{1}$-rich phase at the surface of the aqueous phase is systematically examined according to the wetting coefficient determined from the experimental results of surface/interfacial tensions. It is found that the iso$\mathrm{C}_{4} \mathrm{E}_{1}$-rich phase exhibits a sequence of wetting transitions, nonwetting $\rightarrow$ partial wetting $\rightarrow$ complete wetting, at the water surface in the water + iso- $\mathrm{C}_{4} \mathrm{E}_{1}$ system along with increasing temperature. On the other hand, the iso- $\mathrm{C}_{4} \mathrm{E}_{1}$-rich phase undergoes a wetting transition from partial wetting to nonwetting at the surface of the aqueous phase by increasing the system pressure at a fixed temperature near the lower critical solution temperature (LCST) of the closed-loop miscibility gap in the water + iso- $\mathrm{C}_{4} \mathrm{E}_{1}$ system.
\end{abstract}

\section{Introduction}

Consider a system of three phases $\alpha, \beta$, and $\gamma$ coexisting, and the densities of these three phases are in the order $\rho_{\alpha}<\rho_{\beta}$ $<\rho_{\gamma}$, as schematically shown in Figure $1 \mathrm{c}^{\prime}$. When a small amount of the middle $\beta$ phase is put onto the interface between the lower $\gamma$ phase and the upper $\alpha$ phase, three different wetting behaviors of the $\beta$ phase are possible. They can be distinguished by the wetting coefficient, defined as

$$
W=\left(\sigma_{\alpha \beta}-\sigma_{\alpha \gamma}\right) / \sigma_{\beta \gamma}
$$

The symbol $\sigma_{i j}$ stands for the interfacial tension of the interface separating $i$ and $j$ phases. On the basis of the wetting coefficient $W$, the wetting behavior can then be classified into three categories:

(a) $W=1$. That is a nonwetting $\beta$ phase at the $\alpha-\gamma$ interface, as shown in Figure 1a. In this case, the Antonow's rule ${ }^{1}$ is applied in the form $\sigma_{\alpha \beta}=\sigma_{\alpha \gamma}+\sigma_{\beta \gamma}$. When the system has a substantial amount of the $\beta$ phase, a small amount of the $\gamma$ phase would intrude at the interface separating the $\alpha$ and $\beta$ phases to exhibit complete wetting behavior at the $\alpha-\beta$ interface, as shown in Figure 1a'.

(b) $1>W>-1$. That is a partial wetting $\beta$ phase at the $\alpha-\gamma$ interface, as shown in Figure 1b. The relationship between the three tensions is $\left(\sigma_{\alpha \beta}-\sigma_{\beta \gamma}\right)<\sigma_{\alpha \gamma}<\left(\sigma_{\alpha \beta}+\sigma_{\beta \gamma}\right)$, which follows the Neumann's inequality. ${ }^{2}$

(c) $W=-1$. That is a complete wetting $\beta$ phase at the $\alpha-\gamma$ interface, as shown in Figure 1c. In this case the tensions obey the Antonow's rule, ${ }^{1} \sigma_{\alpha \beta}+\sigma_{\beta \gamma}=\sigma_{\alpha \gamma}$.

A wetting transition is an interfacial phase transition from partial wetting to complete wetting (or nonwetting), and vice versa. Such a transition had been experimentally observed in some binary, ${ }^{3}$ ternary, ${ }^{4}$ and quaternary systems. ${ }^{5}$ In addition, it was found that the wetting transition of the water + oil +

* To whom correspondence should be addressed. E-mail: ljchen@ntu.edu.tw.

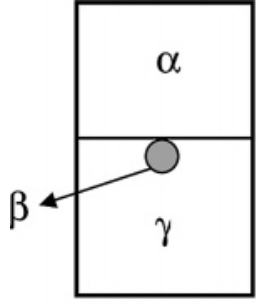

(a)

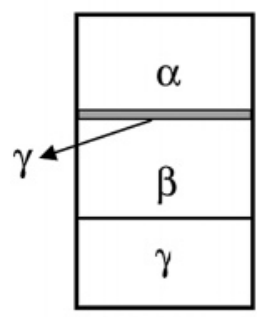

$\left(a^{\prime}\right)$

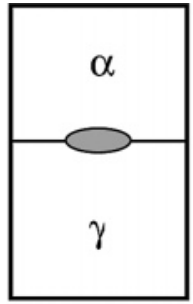

(b)

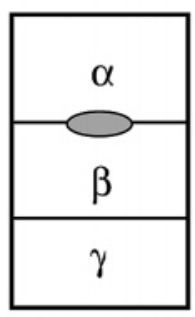

$\left(b^{\prime}\right)$

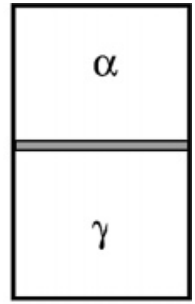

(c)

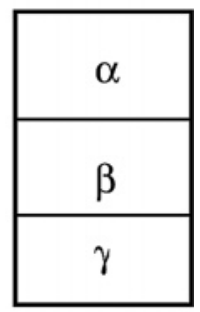

$\left(\mathrm{c}^{\prime}\right)$
Figure 1. Wetting behavior at a fluid-fluid interface: (a) a nonwetting $\beta$ phase at the $\alpha-\gamma$ interface; (a') a complete wetting $\gamma$ phase at the $\alpha-\beta$ interface; (b) a partial wetting $\beta$ phase at the $\alpha-\gamma$ interface; (b') a partial wetting $\gamma$ phase at the $\alpha-\beta$ interface; (c) a complete wetting $\beta$ phase at the $\alpha-\gamma$ interface; and ( $\mathrm{c}^{\prime}$ ) a thick layer of $\beta$ phase separating the $\alpha$ and $\gamma$ phases. The expected condition for only a small amount of the $\beta$ phase at the $\alpha-\gamma$ interface is shown in the upper row (panels $\mathrm{a}, \mathrm{b}$, and $\mathrm{c}$ ), and the condition for a substantial amount of the $\beta$ phase is shown in the lower row (panels $a^{\prime}, b^{\prime}$, and $c^{\prime}$ ). The thickness of the $\gamma$ phase wetting layer in panel $\mathrm{a}^{\prime}$ is exaggerated for illustration.

amphiphile $\mathrm{C}_{i} \mathrm{E}_{j}$ system in the three-liquid-phase-coexisting region occurs as the system is driven to either one of its critical end points by varying the temperature ${ }^{6}$ by varying the amphiphilicity, ${ }^{7}$ by tuning the salinity ${ }^{8}$ and by varying the oil chain length. ${ }^{9}$ (The symbol $\mathrm{C}_{i} \mathrm{E}_{j}$ is the abbreviation of a nonionic polyoxyethylene alcohol $\mathrm{C}_{i} \mathrm{H}_{2 i+1}\left(\mathrm{OCH}_{2} \mathrm{CH}_{2}\right)_{j} \mathrm{OH}$.)

In fact, there are a tremendous number of binary mixtures accessible to experiments. However, only a small subset of 


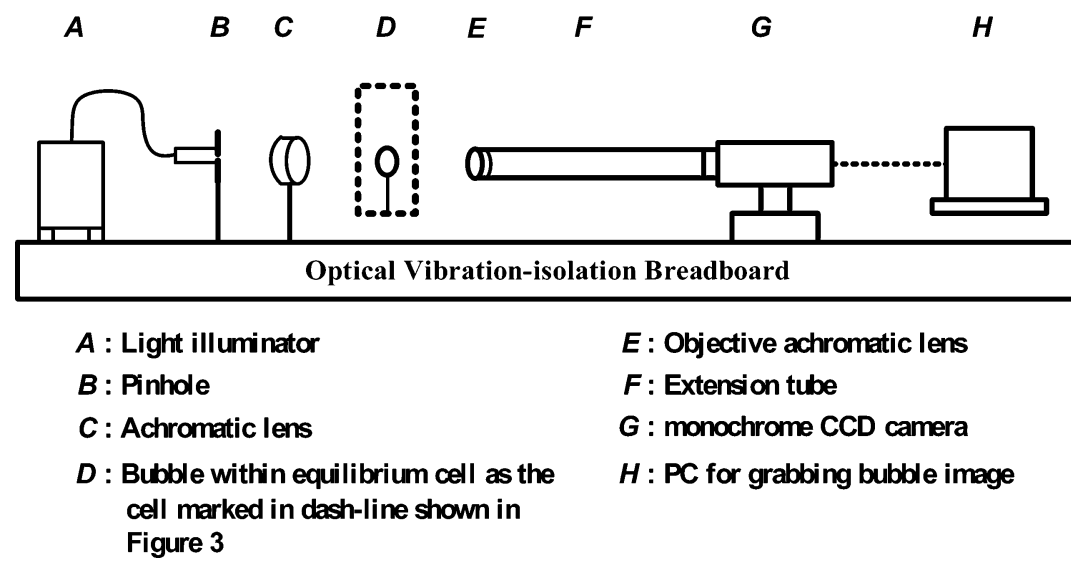

Figure 2. The schematic setup of the pendant bubble/drop tensiometry.

possible binary mixtures has been experimentally observed to study their interfacial wetting behavior. More precisely, most of the experimental studies of binary mixtures in the literature ${ }^{3,10}$ belong to type II or type III mixtures of van Konynenburg and Scott. ${ }^{11}$ Recently, the wetting behavior for type VI mixtures of van Konynenburg and $\mathrm{Scott}^{11}$ had been explored experimentally. ${ }^{12}$

In this study, the wetting transitions induced by temperature and by pressure are carefully examined in the binary water + ethylene glycol monoisobutyl ether (iso- $\mathrm{C}_{4} \mathrm{E}_{1}$ ) mixture, which belongs to type VI mixtures of van Konynenburg and Scott. ${ }^{11}$ It should be noted that, to the best of our knowledge, the pressure-induced wetting transition has never been experimentally verified.

\section{Experiments}

Materials. Ethylene glycol monoisobutyl ether (iso- $\mathrm{C}_{4} \mathrm{E}_{1}$, $>99 \%$ ) was purchased from Merck Chemical Co. and used as received. Water was purified by double distillation followed by a PURELAB Maxima Series (ELGA LabWater) purification system with the resistivity always better than $18.2 \mathrm{M} \Omega-\mathrm{cm}$.

Apparatus. A homemade pendant bubble/drop tensiometer was used to measure the interfacial tension between two coexisting liquid phases and the surface tensions of both liquid phases against helium. The schematic setup of the tensiometer is shown in Figure 2. This device was mainly composed of a parallel light generator including a light source (model 190, fiber optical illuminator, Dolan-Jenner), a diffuser, a pinhole, an achromatic lens, a solid-state monochrome charge-coupled device (CCD) camera (XC-ST70, Sony), and a computer equipped with an image frame grabber (DT3155 MACH Series, Data Translation). The bubble (or liquid drop) image on the active area of the camera was magnified approximately $1 \times$ (or $1.8 \times$ ) for surface (or interfacial) tension measurement. The frame grabber digitized an image into $640 \times 480$ pixels with an 8-bit resolution of 256 gray levels. The bubble (or liquid drop) forming system was composed of a precision syringe pump (model 100DM, ISCO Inc.) and stainless steel needles. Two needles (Hamilton Co.) of different diameters, gauge 17 [1.07 mm inner diameter (i.d.); $1.47 \mathrm{~mm}$ outer diameter (o.d.)] and gauge 27 (0.21 mm i.d.; $0.41 \mathrm{~mm}$ o.d.) were equipped at the bottom of the equilibrium cell for, respectively, the surface and interfacial tension measurements. The glass liquid level gauge (series 20 of JERGUSON Gage \& Valve Co.) was applied as the equilibrium cell. The sample mixture was held in the equilibrium cell, which was housed in a thermostat (TV4000, Tamson Labovisco, Holland). The temperature stability of the thermostat was better than $\pm 0.05 \mathrm{~K}$. The density for each phase was measured by using a vibrating-tube densiometer (DAM 60/ 512, Anton Paar, Austria) directly connected to the equilibrium cell through the pipeline and the liquid pump (LDC analytical minipump). We were able to measure the density online. The composition for each phase was measured by using an online gas chromatography (model 9800, China Chromatography, Taiwan). The detailed layout of the experimental apparatus and the piping is schematically illustrated in Figure 3.

Procedures. The equilibrium cell and all the pipelines were vacuumed by a vacuum pump (GCD-050XA, Sinku Kiko Co., Ltd, Japan). About $100 \mathrm{~mL}$ of degassed sample mixture with a proper composition $\left(\sim 0.3\right.$ mass fraction of iso- $\left.\mathrm{C}_{4} \mathrm{E}_{1}\right)$ was automatically sucked into the equilibrium cell through the pipelines by pressure difference. The inert gas helium of $99.99 \%$ purity was then injected into the system to maintain the system pressure at a prescribed value, as indicated by a digital pressure gauge (DPI280/PDCR330, Druck Limited). The liquid sample in the equilibrium cell was circulated by the liquid pump for at least $2 \mathrm{~h}$ to ensure thorough mixing. The equilibration was established for at least several hours up to 1 day. When the equilibrium was reached, both liquid phases in the equilibrium cell were clear and transparent with sharp and mirrorlike interfaces.

Note that a stainless steel sample cylinder was assembled right beside the equilibrium cell, as shown in Figure 3, for adjusting the level of the interface separating the iso- $\mathrm{C}_{4} \mathrm{E}_{1}$-rich and aqueous phases in the equilibrium cell to perform the pendant bubble/drop method. The Hamilton needle for the pendant bubble/drop measurement was installed at the bottom of the equilibrium cell. Once the sample mixture was input, the whole needle was immersed in the lower aqueous phase after the equilibration. First we performed the surface tension measurement for the lower aqueous phase. Then we had to pump a certain amount of the lower aqueous phase from the equilibrium cell to the sample cylinder to lower the level of the interface separating the iso- $\mathrm{C}_{4} \mathrm{E}_{1}$-rich and aqueous phases, that is to ensure that the tip of the needle was right in the middle of the upper iso- $\mathrm{C}_{4} \mathrm{E}_{1}$-rich phase for further surface tension measurements for the upper iso- $\mathrm{C}_{4} \mathrm{E}_{1}$-rich phase.

The precision syringe pump (model 100DM, ISCO Inc.) was applied to drive the upper fluid phase through the pipelines to form a bubble (or liquid drop) at the selected needle in the lower liquid phase. For each tension, at least 10 different bubbles (or liquid drops) were formed. For each bubble (or liquid drop), at least 10 images were taken for image analysis. An edge detection program was adopted from that of the previous work ${ }^{13}$ to determine the bubble/drop profile from a digitized image. Once a bubble (or a liquid drop) profile was digitized and allocated, 


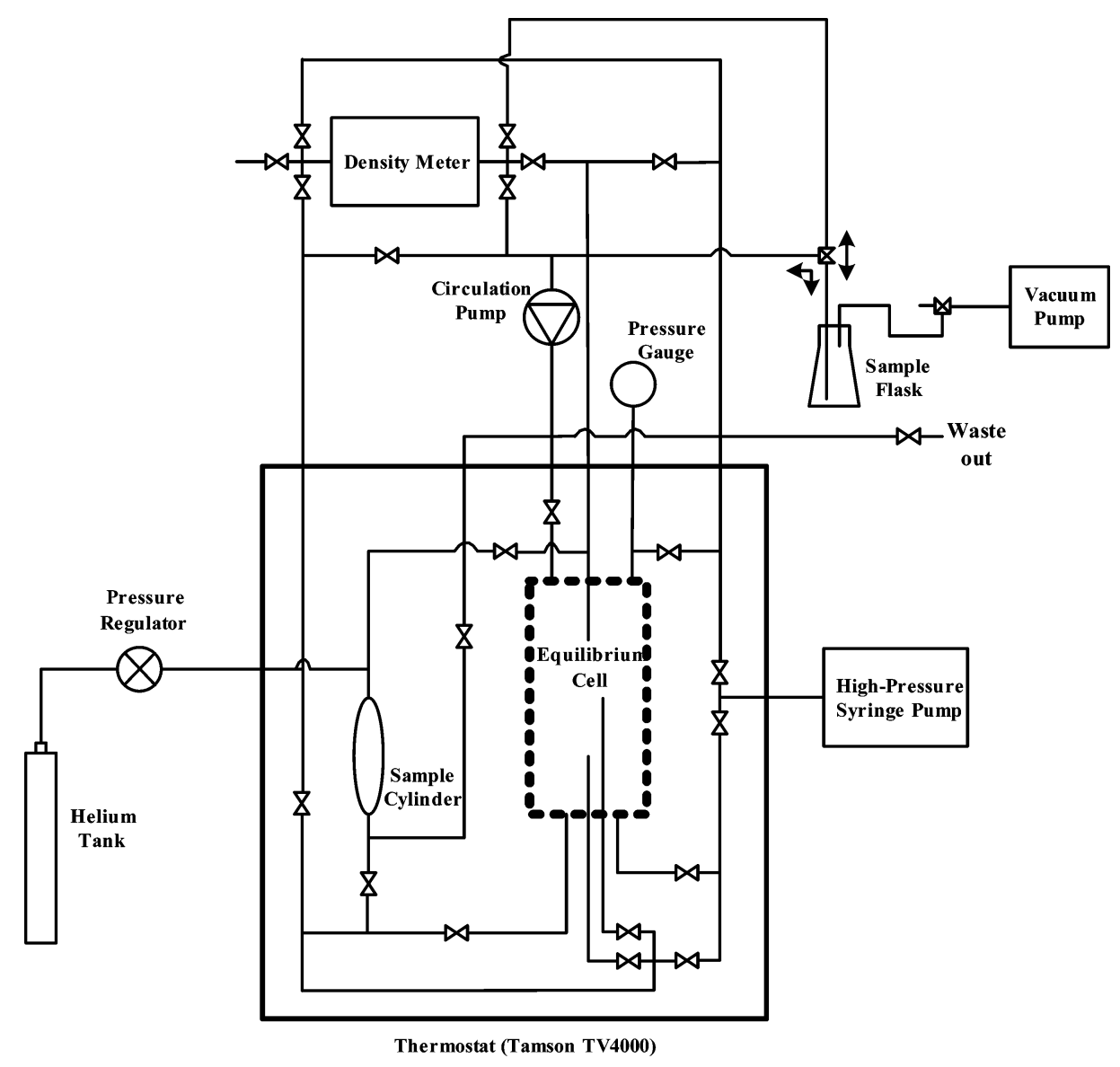

Figure 3. The schematic setup of the experimental apparatus and the bubble/drop forming system.

the selected-plane method was applied to determine the surface (or interfacial) tension by numerically fitting the profile to the Young-Laplace equation. ${ }^{13}$ The experimental uncertainty of the surface/interfacial tension measurements was always better than $0.3 \%$.

\section{Results and Discussion}

It is well understood that the water + iso- $\mathrm{C}_{4} \mathrm{E}_{1}$ system exhibits a closed-loop miscibility gap at 1 bar. The lower critical solution temperature (LCST) and upper critical solution temperature (UCST) of the system are, respectively, 25 and $150{ }^{\circ} \mathrm{C} .{ }^{14} \mathrm{We}$ started with the system to perform its surface/interfacial tensions over its temperature window of closed loop at atmospheric pressure. However, when the temperature was higher than 85 ${ }^{\circ} \mathrm{C}$, the mixture started boiling, and no stable interface was observed any more. Therefore, the inert helium was applied to increase the system pressure up to $10 \mathrm{bar}$, and that suppressed thermal instability to gain stable interfaces for surface/interfacial tension measurements. The $\alpha, \beta$, and $\gamma$ phases defined in Figure $1 \mathrm{c}^{\prime}$ correspond to the inert helium, the iso- $\mathrm{C}_{4} \mathrm{E}_{1}$-rich phase, and the aqueous phase, respectively, in the water + iso- $\mathrm{C}_{4} \mathrm{E}_{1}$ system.

The surface/interfacial tensions for the water + iso- $\mathrm{C}_{4} \mathrm{E}_{1}$ mixture at 10 bar were measured by the homemade pendant drop/bubble tensiometer between UCST and LCST of the system. The experimental results of the surface tensions of the iso- $\mathrm{C}_{4} \mathrm{E}_{1}$-rich phase $\left(\sigma_{\alpha \beta}\right)$ and of the aqueous phase $\left(\sigma_{\alpha \gamma}\right)$ are given in Figure 4, parts a and $b$, respectively. Both surface tensions decrease along with increasing temperature. Figure $4 \mathrm{c}$ shows the variation of experimental results of the interfacial tension between the $\beta$ and $\gamma$ phase $\left(\sigma_{\beta \gamma}\right)$ as a function of temperature ranging from its LCST to UCST. To start with $\sigma_{\beta \gamma}$ $=0$ at LCST, the interfacial tension $\sigma_{\beta \gamma}$ increases along with increasing temperatures, reaches to a maximum around $90{ }^{\circ} \mathrm{C}$, then decreases along with further increasing temperatures, and finally $\sigma_{\beta \gamma}=0$ at UCST.

The wetting behavior of the $\beta$ phase at the $\alpha-\gamma$ interface could be simply verified by the wetting coefficient. Figure 5 illustrates the variation of the wetting coefficient as a function of temperature at 10 bar. The wetting behavior of the $\beta$ phase at the $\alpha-\gamma$ interface for the water + iso- $\mathrm{C}_{4} \mathrm{E}_{1}$ system is then classified into three regimes according to the wetting coefficient. (1) When the temperature is below $31.5^{\circ} \mathrm{C}, W=1$. The $\beta$ phase exhibits nonwetting behavior. (2) When the temperature falls into the range from 31.5 to $107.0{ }^{\circ} \mathrm{C}, 1>W>-1$. The $\beta$ phase exhibits partial wetting behavior. (3) When the temperature is above $107.0{ }^{\circ} \mathrm{C}, W=-1$. The $\beta$ phase exhibits complete wetting behavior.

Note that there are two wetting transitions in this system. To start with the water + iso- $\mathrm{C}_{4} \mathrm{E}_{1}$ system at $70{ }^{\circ} \mathrm{C}$, the $\beta$ phase exhibits partial wetting behavior at the surface of the aqueous phase. A wetting transition from partial wetting to nonwetting occurs at $31.5{ }^{\circ} \mathrm{C}$ while the system is brought to approach its LCST. In the other direction, while the system is driven to approach its UCST, another wetting transition from partial wetting to complete wetting happens at $107.0^{\circ} \mathrm{C}$. In other words, a sequence of wetting transition, nonwetting $\rightarrow$ partial wetting $\rightarrow$ complete wetting, is observed in the water + iso- $\mathrm{C}_{4} \mathrm{E}_{1}$ system with increasing temperatures under 10 bar. It should be noted that exactly the same sequence of wetting transition was also observed in the water $+\mathrm{C}_{4} \mathrm{E}_{1}$ system by simply varying the temperature.

These experimental results verify the conjecture of Kahlweit and Busse ${ }^{12 \mathrm{~d}}$ on the occurrence of the sequence of wetting transition in the binary water $+\mathrm{C}_{i} \mathrm{E}_{j}$ mixture. These authors 
(a)

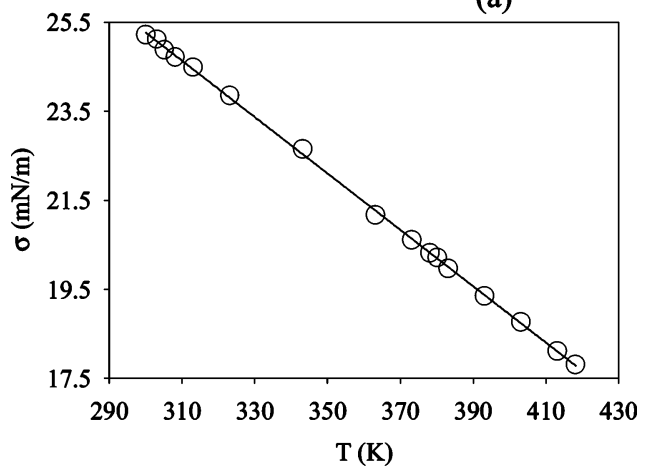

(b)

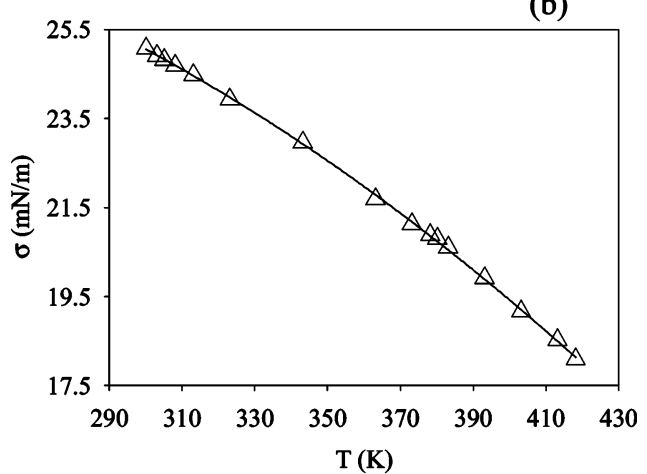

(c)

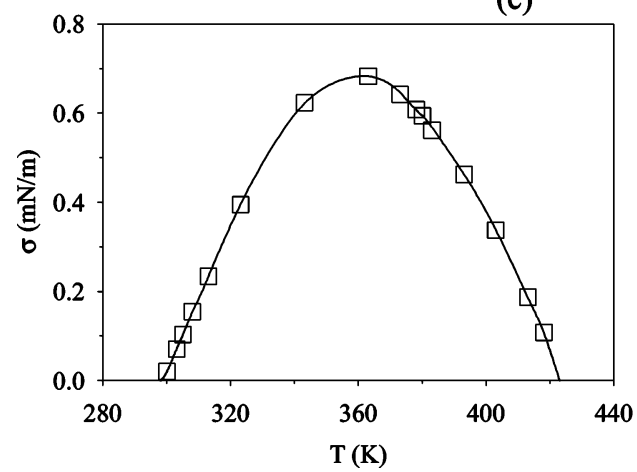

Figure 4. Variation of surface/interfacial tensions as a function of temperature for the water $+\mathrm{C}_{4} \mathrm{E}_{1}$ system at 10 bar: (a) $\sigma_{\alpha \beta}$, (b) $\sigma_{\alpha \gamma}$, and (c) $\sigma_{\beta \gamma}$

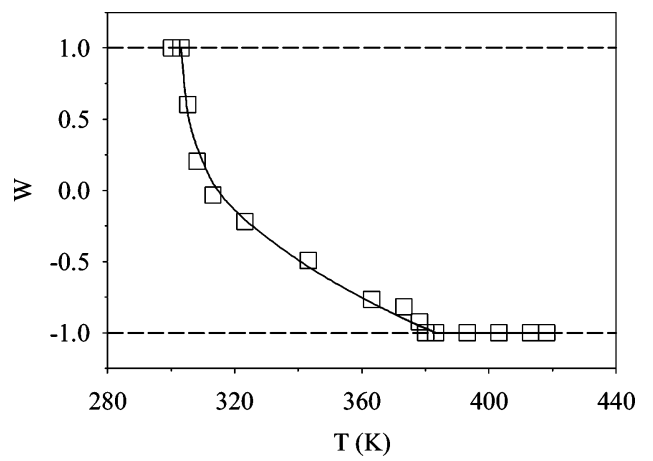

Figure 5. Variation of wetting coefficients as a function of temperature for the water + iso- $\mathrm{C}_{4} \mathrm{E}_{1}$ system at 10 bar.

carefully investigated the wetting behavior of the water $+\mathrm{C}_{8} \mathrm{E}_{j}$ mixtures at $298.15 \mathrm{~K}$ by direct observation of the contact angle $\theta$ of the suspended lens of the $\mathrm{C}_{8} \mathrm{E}_{j}$-rich phase at the air-water interface. ${ }^{12 \mathrm{~d}}$ The contact angle $\theta$ increases along with an increase in the number of oxyethylene groups $j$ from 0 to 3 stepwise. Based on this phenomenological argument, Kahlweit and Busse ${ }^{12 \mathrm{~d}}$ conjectured that the $\mathrm{C}_{i} \mathrm{E}_{j}$-rich phase in the water +

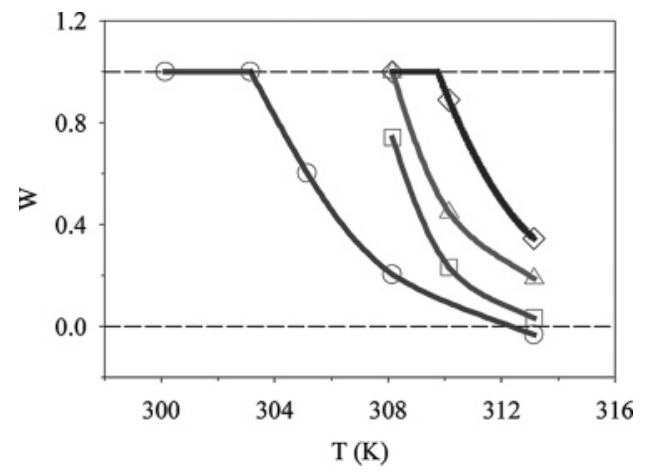

Figure 6. Variation of wetting coefficients as a function of temperature for the water + iso- $\mathrm{C}_{4} \mathrm{E}_{1}$ system at four different pressures: 10 bar (circle); 40 bar (square); 70 bar (triangle); 100 bar (diamond).

$\mathrm{C}_{8} \mathrm{E}_{j}$ mixtures exhibits a sequence of wetting transitions from nonwetting to partial wetting to complete wetting at the airwater interface along with increasing temperature from its LCST to UCST. All these results are consistent with the prediction of the critical point wetting theory of $\mathrm{Cahn}^{15}$ and of Ebner and Saam. ${ }^{16}$

To examine the pressure effect on wetting behavior, the surface/interfacial tensions for water + iso- $\mathrm{C}_{4} \mathrm{E}_{1}$ mixtures were measured near its $\operatorname{LCST}\left(35,37\right.$, and $\left.40{ }^{\circ} \mathrm{C}\right)$ at four different pressures, 10, 40, 70, and 100 bar. The wetting coefficients for the water + iso- $\mathrm{C}_{4} \mathrm{E}_{1}$ system were then determined from the surface/interfacial tensions. Figure 6 shows the variation of the wetting coefficient as a function of temperature at four different pressures. Figure 6 illustrates obviously that the wetting coefficient drops substantially right after the occurrence of the transition from nonwetting to partial wetting at each pressure by simply increasing temperatures. For the system at a fixed pressure, the wetting transition temperature increases along with increasing pressures.

It has been experimentally ${ }^{17}$ shown for the water $+\mathrm{C}_{4} \mathrm{E}_{1}$ system the closed loop phase diagram can be visualized as a vertical section (constant pressure) through a "nose" in composition-temperature-pressure space, as schematically demonstrated in Figure 7a. The closed loop (temperature-composition plane) shrinks with increasing pressures, as schematically illustrated in Figure 7b, and disappears completely at a definite pressure. On the other hand, the binary system $\mathrm{H}_{2} \mathrm{O}+\mathrm{C}_{i} \mathrm{E}_{j}$ exhibits a dome-shaped phase behavior at a fixed temperature that can be visualized as a horizontal section (constant temperature) through the "nose" schematically illustrated in Figure 7a. There exists a critical pressure for the dome-shaped phase behavior at a fixed temperature, as schematically shown in Figure $7 \mathrm{c}$. The dome-shaped phase diagram of pressurecomposition plane enlarges (or shrinks) with increasing temperatures for the lower (or upper) half of the "nose." According to the critical wetting theory, a wetting transition must occur from partial wetting behavior to complete wetting (or nonwetting) behavior as a multiphase-coexisting system is brought close to a critical end point. Therefore, a wetting transition from partial wetting to nonwetting behavior should be observed in the water + iso- $\mathrm{C}_{4} \mathrm{E}_{1}$ system when the system is driven to approach its critical pressure at a fixed temperature near its LCST.

Figure 6 indeed shows that the wetting coefficient increases along with increasing pressures at any fixed temperature. For example, at $35{ }^{\circ} \mathrm{C}$, the wetting coefficients are $0.205,0.742$, 1.00 , and 1.00 for the system at $10,40,70$, and $100 \mathrm{bar}$, respectively. Obviously, there exists a wetting transition pressure between 40 and 70 bar. When the temperature slightly increases up to $37^{\circ} \mathrm{C}$, the wetting transition pressure is larger than 100 

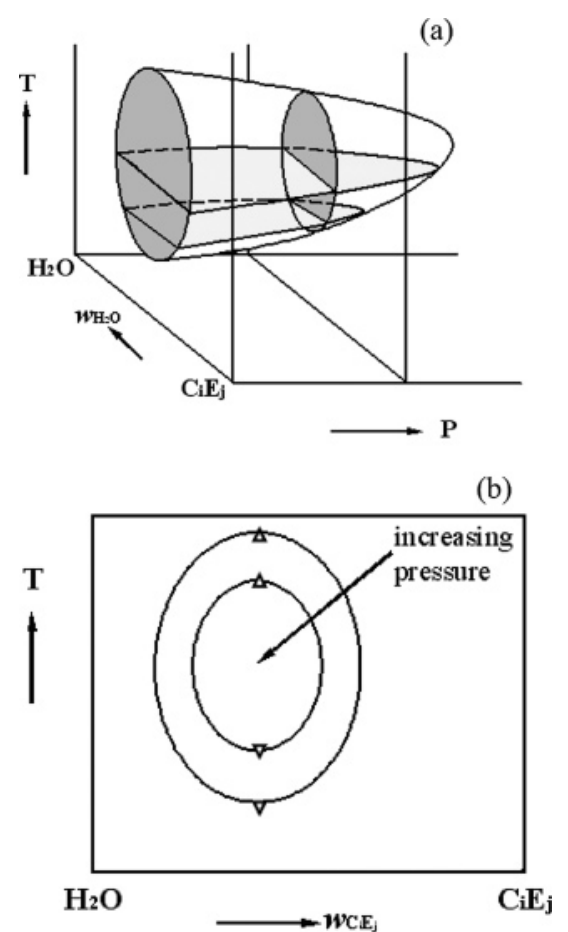

(c)

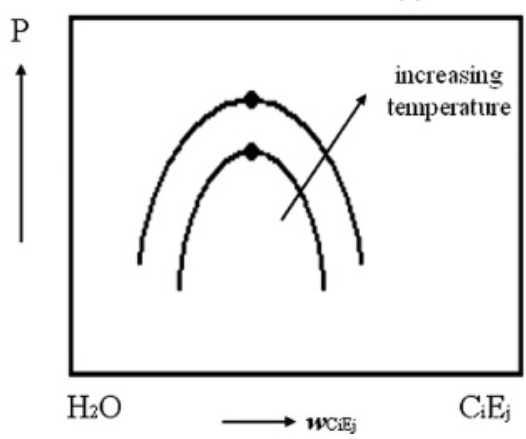

Figure 7. Pressure effect on immiscibility phenomena for the binary water $+\mathrm{C}_{i} \mathrm{E}_{j}$ system: (a) schematic plot of temperature-pressurecomposition three-dimensional phase diagram; (b) shrinking of the closed loop (temperature-composition plane) by increasing the system pressures. The closed loop can be considered as a vertical section (constant pressure) through the 'nose' in temperature-pressurecomposition space. Panel c shows the enhancement of the dome-shaped phase diagram of pressure-composition plane with increasing temperatures for the lower half of the "nose."

bar, since the $\beta$ phase exhibits partial wetting behavior $(W=$ 0.89) at the $\alpha-\gamma$ interface at 100 bar. Thus, the wetting transition pressure increases along with increasing temperatures.

It is plausible to conjecture that another wetting transition from partial wetting to complete wetting can also be induced by increasing pressures at a fixed temperature near the UCST of the system. However, the UCST is higher than $100{ }^{\circ} \mathrm{C}$, and our homemade pendant drop/bubble tensiometer cannot endure high pressures at such high temperatures.

In summary, the surface/interfacial tension measurements were performed over the wide temperature and pressure ranges of the water + iso- $\mathrm{C}_{4} \mathrm{E}_{1}$ system. On the basis of the wetting coefficient data, it is found that the iso- $\mathrm{C}_{4} \mathrm{E}_{1}$-rich phase exhibits a sequence of wetting transitions, nonwetting $\rightarrow$ partial wetting $\rightarrow$ complete wetting, at the surface of the aqueous phase by increasing the system temperature. On the other hand, the wetting transition from partial wetting to nonwetting (or complete wetting) is induced by increasing pressures at a fixed temperature near the LCST (or UCST) of the system.

Acknowledgment. This work was supported by the National Science Council of Taiwan.

\section{References and Notes}

(1) Antonow, G. N. J. Chim. Phys. Phys. Chim. Biol. 1907, 5, 372

(2) Buff, F. P. Encyclopedia of Physics; Flugge, S. Ed., Springer: Berlin, Germany, 1960; Vol. 10, pp 298-299.

(3) (a) Moldover, M. R.; Cahn, J. W. Science 1980, 207, 1073. (b) Pohl, D. W.; Goldburg, W. I. Phys. Rev. Lett. 1982, 48, 1111. (c) Schmidt, J. W.; Moldover, M. R. J. Chem. Phys. 1983, 79, 379. (d) Bonn, D.; Hellay, H.; Wegdam, G. H. Phys. Rev. Lett. 1992, 69, 1975.

(4) (a) Kahlweit, M.; Busse, G.; Haase, D.; Jen, J. Phys. Rev. A: At. Mol., Opt. Phys. 1988, 38, 1395. (b) Carrillo, E.; Talanquer, V.; Costas, M. J. Phys. Chem. 1996, 100, 5888.

(5) Estrada-Alexanders, A.; Garcia-Valenzuela, A.; Guzman, F. J. Phys. Chem. 1991, 95, 219.

(6) (a) Aratono, M.; Kahlweit, M. J. Chem. Phys. 1991, 95, 8578. (b) Chen, L.-J.; Jeng, J.-F.; Robert, M.; Shukla, K. P. Phys. Rev. A: At., Mol., Opt. Phys. 1990, 42, 4716. (c) Chen, L.-J.; Yan, W.-J. J. Chem. Phys. 1993, 98, 4830. (d) Chen, L.-J.; Yan, W.-J.; Hsu, M.-C.; Tyan, D.-L. J. Phys. Chem. 1994, 98, 1910. (e) Chen, L.-J.; Lin, S.-Y.; Xyu, J.-W. J. Chem Phys. 1996, 104, 225. (f) Smith, D. H.; Covatch, G. L. J. Chem. Phys. 1990, 93, 6870

(7) Kahlweit, M.; Strey, R.; Busse, G. Phys. Rev. E: Stat. Phys., Plasmas, Fluids, Relat. Interdiscip. Top. 1993, 47, 4197.

(8) (a) Chen, L.-J.; Hsu, M.-C. J. Chem. Phys. 1992, 97, 690. (b) Chen, L.-J.; Hsu, M.-C.; Lin, S.-T.; Yang, S.-Y. J. Phys. Chem. 1995, 99, 4687.

(9) (a) Kahlweit, M.; Busse, G. J. Phys. Chem. B 2000, 104, 4939. (b) Chen, L.-J.; Chiu, C.-D.; Shau, F.-S.; Cheng, W.-J.; Wu, J.-G. J. Phys. Chem. B 2002, 106, 12782.

(10) (a) Ragil, K.; Meunier, J.; Broseta, D.; Indekeu, J. O.; Bonn, D. Phys. Rev. Lett. 1996, 77, 1532. (b) Ross, D.; Bonn, D.; Meunier, J. Nature 1999, 400, 737. (c) Bertrand, E.; Dobbs, H.; Broseta, D.; Indekeu, J. O.; Bonn, D.; Meunier, J. Phys. Rev. Lett. 2000, 85, 1282.

(11) van Konynenburg, P. H.; Scott, R. L. Phios. Trans. R. Soc. London, Ser. A 1980, 298, 495

(12) (a) Yeh, M.-C.; Lin, P.-C.; Chen, L.-J. J. Phys. Chem. B 2004, 108, 9955. (b) Wu, C.-K.; Chen, L.-J. J. Chem. Phys. 2005, 123 (8), 084506. (c) Wu, C.-K.; Chen, L.-J. Langmuir 2005, 21, 6883. (d) Kahlweit, M.; Busse, G. J. Chem. Phys. 1989, 15, 1339.

(13) (a) Yeh, M.-C.; Chen, L.-J.; Lin S.-Y.; Hsu, C.-T. J. Chin. Inst. Chem. Eng. 2001, 32, 109. (b) Lin, S.-Y.; Chen, L.-J.; Xyu, J.-W.; Wang, W.-J. Langmuir 1995, 11, 4159. (c) Lin, S.-Y.; Chen, L.-J.; Wang, W.-J.; Lin, L.-W. Colloids Surf., A 1996, 114, 31.

(14) Cox, H. L.; Cretcher, L. H. J. Am. Chem. Soc. 1926, 48, 451.

(15) Cahn, J. W. J. Chem. Phys. 1977, 66, 3667.

(16) Ebner, C.; Saam, W. F. Phys. Rev. Lett. 1977, 38, 1486.

(17) (a) Schneider, G. M. Z. Phys. Chem. (Neue Folge) 1963, 37, 333. (b) Kahlweit, M.; Strey, R. Angew. Chem., Int. Ed., Engl. 1985, 24, 654 